Disclosure of Interest: None declared

DOI: 10.1136/annrheumdis-2018-eular.3343

\section{OP0350 DEPRESSION AND ANXIETY IN AN EARLY RHEUMATOID ARTHRITIS INCEPTION COHORT. ASSOCIATIONS WITH EPIDEMIOLOGICAL, SOCIOECONOMIC AND DISEASE FEATURES}

G.E. Fragoulis, J. Cavanagh, M.H. Derakhshan, C. Paterson, D. Porter, I. B. McInnes, S. Siebert. Institute of Infection, Immunity and Inflammation, University of Glasgow, Glasgow, UK

Background: Co-morbid depression and anxiety occur in the context of rheumatoid arthritis (RA). Their characteristics, including associations with RA features, have not been examined previously in an early RA inception cohort with longitudinal follow up data.

Objectives: To examine the frequency of anxiety and depression in patients with early RA, over time and to explore associations with epidemiological, socioeconomic and disease-related features.

Methods: The Scottish Early Rheumatoid Arthritis (SERA) inception cohort recruited newly diagnosed RA patients (fulfilling ACR-EULAR 2010 criteria) followed-up thereafter every 6 months. Pre-specified clinical, laboratory and psychosocial features, including anxiety and depression scores (measured by the hospital anxiety and depression scale; score range: 0-21 for each one), were recorded at baseline and at follow-up. Non-parameteric tests and logistic regression models were used to examine the nature and magnitude of associations. Results: Data from 848 RA patients were available. Frequency of anxiety and depression at baseline was $19.0 \%$ and $12.2 \%$ with a reduction at month $12 \%$ to $13.4 \%$ and $8.2 \%(p=0.004$ and $p=0.01$, respectively). Anxiety and depression scores were correlated with DAS28 at all time points, including baseline (all $\mathrm{p}<0.0001$ ). Change in DAS28 (final-baseline) was correlated with change in depression and anxiety scores at month $6(p<0.0001, r=0.265$ and $p=0.001$, $r=0.230)$ and $12(p<0.0001, r=0.288$ and $p<0.0001, r=0.217)$. In univariate analyses, anxiety and depression scores were associated with various features, at different time points (table 1). CRP was highly associated with depression but not anxiety scores at all time points, with change in CRP correlating with change in depression scores (month6; $p<0.0001, r=0.185$ and month12; $p<0.0001$, $r=0.302$ ). Multivariable analysis indicated that anxiety score at baseline was associated with female gender $(p=0.01$, Beta $=0.133)$, younger age $(p=0.007$, Beta $=-0.181)$ and patient global assessment score (PGA) $(p<0.0001$, Beta=0.201), and at month 6 and 12 with low BMI (month $6, p=0.024$, Beta $=-0.091$; month 12, $\mathrm{p}=0.002$, Beta $=-0.139$ ), PGA (month $6, p<0.0001$, Beta $=0.222$; month 12, $\mathrm{p}<0.0001$, Beta $=0.248$ ) and baseline anxiety scores (month 6, $\mathrm{p}<0.0001$, Beta=0.623; month 12, $\mathrm{p}<0.0001$, Beta=0.586). For depression scores, multivariable analysis indicated association at baseline with PGA $(p<0.0001$, Beta $=0.286)$ and at month 6 and 12 with PGA (month $6, p<0.0001$, Beta $=0.306$; month 12, $p<0.0001$, Beta $=0.320$ ), CRP levels (month $6, p=0.006$, Beta $=0.150$; month 12, $p=0.002$, Beta $=0.171$ ) and baseline depression (month 6 , $\mathrm{p}<0.0001$, Beta $=0.422$; month $12, \mathrm{p}<0.0001$, Beta=0.356) and anxiety scores (month 6, $\mathrm{p}<0.0001$, Beta $=0.189$; month 12, $\mathrm{p}=0.008$, Beta $=0.170$ ).

Abstract OP0350 - Table 1 Variables associating with high anxiety and/or depression score at baseline and at month 6 and 12. 1) ANOVA test, compared to employed and to retired individuals, 2) patient global VAS used for DAS28 calculation. 3) average alcohol units week. NA: not applicable, BMI: body mass index, IM: intramuscular. ^by mouth, *intramuscular

\begin{tabular}{|c|c|c|c|c|c|c|}
\hline \multicolumn{7}{|c|}{ Anxietyscore } \\
\hline & \multicolumn{2}{|c|}{ Baseline } & \multicolumn{2}{|c|}{6 months } & \multicolumn{2}{|c|}{12 months } \\
\hline Factors/confounders & p value & $r$ & p value & $r$ & Pvalue & $r$ \\
\hline Age & $<0.001$ & -0.187 & $<0.001$ & -0.158 & 0.001 & -0.131 \\
\hline BMI & & & & & 0.044 & 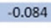 \\
\hline Female gender & 0.002 & NA & & & & \\
\hline Smoking & & & 0.008 & NA & 0.039 & NA \\
\hline Unemployment ${ }^{1}$ & 0.001 & NA & $<0.001$ & NA & $<0.001$ & NA \\
\hline Anxiety baseline & & & $<0.001$ & 0.691 & $<0.001$ & 0.632 \\
\hline Depression baseline & & & $<0.001$ & 0.507 & $<0.001$ & 0.443 \\
\hline DAS28 baseline & & & $<0.001$ & 0.175 & 0.020 & 0.114 \\
\hline Patient global VAS ${ }^{2}$ & $<0.001$ & 0.210 & $<0.001$ & 0.397 & $<0.001$ & 0.387 \\
\hline \multicolumn{7}{|c|}{ Depression score } \\
\hline & \multicolumn{2}{|c|}{ Baseline } & \multicolumn{2}{|c|}{6 months } & \multicolumn{2}{|c|}{12 months } \\
\hline Factors/confounders & p value & r & p value & $r$ & Pvalue & $r$ \\
\hline Age & 0.004 & -0.101 & & & & \\
\hline BMI & 0.037 & 0.075 & & & & \\
\hline Alcohol units ${ }^{3}$ & 0.002 & -0.107 & & & & \\
\hline Smoking & & & 0.021 & NA & 0.002 & NA \\
\hline Unemployment & 0.001 & NA & $<0.0001$ & NA & $<0.0001$ & NA \\
\hline Anti-CCP negative & 0.013 & NA & & & & \\
\hline Corticosteroids & & & $0.017^{\wedge}$ & NA & $0.005^{*}$ & NA \\
\hline Anxiety baseline & & & $<0.001$ & 0.488 & $<0.001$ & 0.423 \\
\hline Depression baseline & & & $<0.001$ & 0.602 & $<0.001$ & 0.519 \\
\hline DAS28 baseline & & & $<0.001$ & 0.223 & $<0.0001$ & 0.172 \\
\hline Patient global VAS & $<0.0001$ & 0.328 & $<0.001$ & 0.500 & $<0.001$ & 0.522 \\
\hline ESR & 0.016 & 0.116 & & & 0.029 & 0.130 \\
\hline CRP & $<0.0001$ & 0.163 & $<0.0001$ & 0.152 & 0.004 & 0.112 \\
\hline
\end{tabular}

Conclusions: Depression and anxiety are significant comorbidities at the time of RA diagnosis. While there are also associations with socioeconomic and other variables, the close relationship between CRP and depression provides further support to the already compelling data linking inflammation and depression. Changes in the anxiety and depression scores, in tandem with disease activity over time, requires further investigation.

Disclosure of Interest: None declared

DOI: 10.1136/annrheumdis-2018-eular.7083

SATURDAY, 16 JUNE 2018

\section{Battling hyperinflammation in paediatric rheumatic diseases}

\section{OP0351 FIRST DIAGNOSIS OF UVEITIS IS NOT HIGHER AMONG JUVENILE IDIOPATHIC ARTHRITIS (JIA) PATIENTS RECEIVING ETANERCEPT COMPARED TO METHOTREXATE}

R. Davies ${ }^{1}$, D. De Cock ${ }^{1}$, L. Kearsley-Fleet ${ }^{1}$, A. Ramanan ${ }^{2,3}$, K.L. Hyrich ${ }^{1,4}$, on behalf of British Society for Paediatric and Adolescent Rheumatology Etanercept Cohort Study. ${ }^{1}$ Arthritis Research UK Centre for Epidemiology, Manchester Academic Health Science Centre, University of Manchester, Manchester ${ }^{2}$ Pediatric Rheumatology, University Hospitals Bristol NHS Foundation Trust, ${ }^{3}$ Ophthalmology, School of Clinical Sciences, University of Bristol, Bristol; ${ }^{4}$ National Institute of Health Research Manchester Musculoskeletal Biomedical Research Centre, Manchester Academic Health Science Centre, Manchester University NHS Foundation Trust, Manchester, UK

Background: Uveitis is a common comorbidity among patients with juvenile idiopathic arthritis (JIA), occurring in approximately 1 in $10 \mathrm{JIA}$ patients. Among other risk factors such as early ageat JIA onset, shorter disease duration and oligoarticular subtype, the use of etanercept (ETN) may also increase the risk of developing uveitis. However, previous studies have produced conflicting results, often limited by small sample sizes and limited follow-up time.

Objectives: To determine if patients receiving ETN have a higher risk of developing uveitis for the first time compared to patients receiving methotrexate (MTX). Methods: The study population comprised JIA subjects recruited to the BSPAR ETN Cohort Study at point of starting ETN or MTX. Only patients with no prior history of uveitis were included. This was an on-drug analysis, whereby events were only included if the patient was on ETN or MTX at the time of uveitis onset. Followup began from date of first treatment to first uveitis diagnosis, discontinuation of ETN or MTX, most recent follow-up up to 30/11/16 or death, whichever came first. Crude incidence rates of uveitis per 100 person years (pyears) were calculated. Hazard ratios (HR) comparing risk of uveitis with ETN versus MTX were calculated using propensity adjusted Cox regression.

Results: Of 1517 patients, 1009 were registered to the ETN cohort (all receiving ETN) and 508 to the MTX cohort. ETN patients were older, with longer disease duration, and were less likely to have persistent oligoarthritis. The mean age at uveitis diagnosis was 8 years in the ETN cohort versus 5 years in the MTX cohort. The HR adjusted for age and gender, disease scores, disease duration, baseline steroid use, co-morbidity, ILAR subtype, and ethnicity found a lower risk of developing uveitis inpatients receiving ETN compared to MTX $(0.30,95 \% \mathrm{Cl}(0.10-$ 0.90)) (table 1).

\begin{tabular}{lll} 
Abstract OP0351 - Table 1 & & \\
\hline New onset uveitis & Patients taking MTX & Patients taking EN \\
\hline Subjects (n) & 508 & 1009 \\
Age, median (IQR) & $9(3-13)$ & $11(8-14)$ \\
Gender, \% female & 71 & 69 \\
Disease duration (years), median (IQR) & $1(0-1)$ & $3(1-6)$ \\
CHAQ score, median (IQR) & $0.9(0.3-1.5)$ & $1.0(0.3-1.6)$ \\
ILAR subtype, n (\%) & & \\
Persistent oligoarthritis & $84(17)$ & $53(5)$ \\
Other non-systemic JIA & $424(83)$ & $955(95)$ \\
Person years exposure & 908 & 2471 \\
New diagnosis of uveitis & 18 & 15 \\
Crude incidence rates per 100 pyears & $2.4(1.4-3.9)$ & $0.6(0.3-1.0)$ \\
Unadjusted HR (95\% CI) & ref & $0.24(0.11-0.52)^{*}$ \\
Age \& Gender adjusted HR (95\% Cl) & ref & $0.41(0.18-0.95)^{*}$ \\
Fully adjusted HR (95\% CI) & ref & $0.30(0.10-0.90)^{*}$ \\
\hline
\end{tabular}

Fully adjusted HR $(95 \%$ C $)$

${ }^{*} p<0.05$

Conclusions: Within this cohort of UK children with JIA, a new diagnosis of uveitis is not more common among children receiving ETN compared to MTX, even after taking into account differences inage and disease duration between the cohorts. This is reassuring given there ports of possible increased risk of uveitis among children with JIA receiving ETN. Age appears to be a major influencing factor as patients in the MTX cohort were younger thus at a higher risk of uveitis. For patients with a high eruveitis risk other treatments options could have been 
selected, leading to possible confounding by indication, which in turn makes ETN look protective. Researchers should take these selection biases into account when analysing their results.

Disclosure of Interest: None declared

DOI: 10.1136/annrheumdis-2018-eular.5211

SATURDAY, 16 JUNE 2018

Gut bacteria: the boss of the immune system

\section{OP0352 AN INCREASED PREVALENCE OF PERIODONTAL DISEASE, PORPHYROMONAS GINGIVALIS AND AGGREGATIBACTER ACTINOMYCETEMCOMITANS IN ANTI-CCP POSITIVE INDIVIDUALS AT-RISK OF INFLAMMATORY ARTHRITIS}

K. Mankia ${ }^{1}$, Z. Cheng ${ }^{2}$, T. Do ${ }^{2}$, J. Kang ${ }^{2}$, L. Hunt ${ }^{1}$, J. Meade ${ }^{2}$, V. Clerehugh ${ }^{3}$ A. Speirs ${ }^{3}$, A. Tugnait ${ }^{3}$, E. Hensor ${ }^{4}$, D. Devine ${ }^{2}$, P. Emery ${ }^{1}{ }^{1}$ Rheumatology, Leeds Institute of Rheumatic and Musculoskeletal Medicine and NIHR Leeds Biomedical Research Centre; ${ }^{2}$ Oral Microbiology, University of Leeds; ${ }^{3}$ Leeds Dental Institute, Leeds Teaching Hospitals Trust, ${ }^{4}$ Rheumatology, Leeds Institute of Rheumatic and Musculoskeletal Medicine, Leeds, UK

Background: The prevalence of periodontal disease is increased in RA, and periodontitis is associated with the bacterium Porphyromonas gingivalis $(\mathrm{Pg})$, which can citrullinate arginine residues. ${ }^{1}$ These observations suggest periodontitis may be a key initiator of RA-related autoimmunity. Importantly, clinical periodontal disease, and the relative abundance of periodontal bacteria have not been described in seropositive individuals at risk of developing RA who do not have synovitis.

Objectives: To investigate the prevalence of periodontal disease and the relative abundance of key periodontal bacteria in anti-CCP positive at-risk individuals without synovitis.

Methods: Anti-CCP positive individuals with musculoskeletal symptoms but no clinical synovitis $(\mathrm{CCP}+)$, early RA (RA) patients and healthy controls $(\mathrm{HC})$ were recruited. CCP +underwent a 38 joint ultrasound (US) assessment. Periodontal examination was performed by a dentist; six sites per tooth were assessed for clinical attachment level (CAL), pocket depth (PD) and bleeding on probing (BOP). Periodontal disease sites (PDD) were defined as $C A L>2 \mathrm{~mm}$ and $P D>4 \mathrm{~mm}$. A clinical consensus was agreed for each case by three dentists. DNA, isolated from subgingival plaque from diseased and healthy periodontal sites, was pairend sequenced (Illumina HiSeq3000). Taxonomic and functional profiles were obtained from MG-Rast and differences between groups studied using DESeq2. Mann-Whitney $\mathrm{U}$ tests were used to compare groups and Spearman Rho used for correlations. For metagenomic data, Wald test was used to compare relative abundance.

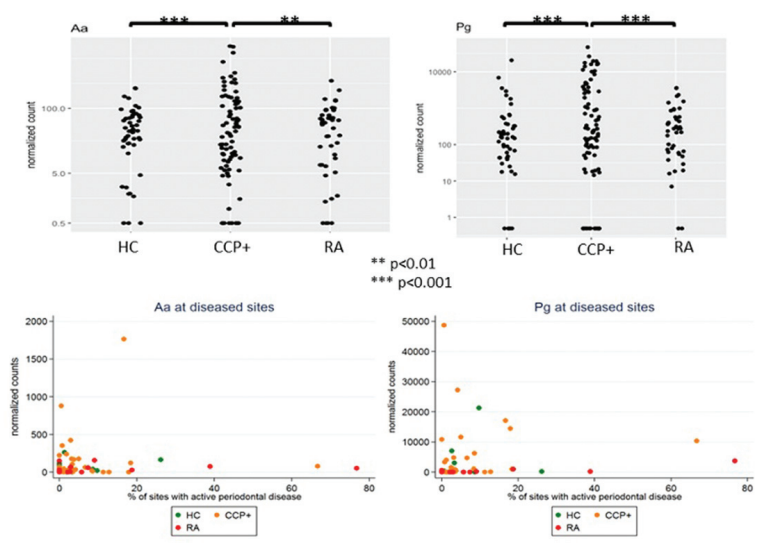

Abstract OP0352 - Figure 1 Relative abundance of Aggregatibacter actinomycetemcomitans $(\mathrm{Aa})$ and Porphyromonas gingivalis $(\mathrm{Pg})$ according to RA status and extent of periodontal disease

Results: $48 \mathrm{CCP}+, 26 \mathrm{RA}$ and $32 \mathrm{HC}$ were recruited. Groups were balanced for age, sex and smoking. All but 2 (96\%) CCP thad no US synovitis (greyscale $\geq 1$ and power Doppler $\geq 1$ ). Dentists classified $73 \% \mathrm{CCP}+, 38 \% \mathrm{HC}$ $(p=0.02)$ and $54 \%$ RA as having clinical periodontitis. The percentage of periodontal sites with $\mathrm{CAL}>2 \mathrm{~mm}, \mathrm{PD}>4 \mathrm{~mm}, \mathrm{BOP}, \mathrm{PDD}$ and active PDD (PDD +BOP) were all greater in $\mathrm{CCP}+$ compared to $\mathrm{HC}(\mathrm{p}<0.05)$ and similar to $\mathrm{RA}$. In nonsmokers, PDD and active PDD were more prevalent in $\mathrm{CCP}+$ compared to $\mathrm{HC}$. Metagenomic data indicated $\mathrm{CCP}$ +had increased relative abundance of both $\mathrm{Pg}$ and Aggregatibacter actinomycetemcomitans $(A a)$ compared to $\mathrm{HC}(\mathrm{p}<0.001)$ and RA $(\mathrm{p}<0.01)$. However, clinical periodontitis was only associated with increased relative abundance of $P g(\mathrm{p}<0.001)$ but not $A a$. Furthermore, the rela tive abundance of $P g$ was associated with the percentage of sites with active PDD in $\mathrm{CCP}+(\mathrm{p}=0.05)$ and $\mathrm{HC}(\mathrm{p}=0.04)$ but this was not seen for Aa (figure 1).

Conclusions: We report an increased prevalence of periodontal disease, $\mathrm{Pg}$ and $A a$ in anti-CCP positive at-risk individuals without synovitis. Interestingly, relative abundance of $\mathrm{Pg}$, but not $\mathrm{Aa}$, was associated with periodontitis, suggesting potential mechanistic differences that require further exploration. These data support the concept that periodontal inflammation and periodontopathic bacteria may both be important in the initiation of RA-related autoimmunity.

\section{REFERENCE:}

[1] Lundberg K. Nat Rev Rheumatol 2010

Disclosure of Interest: None declared

DOI: 10.1136/annrheumdis-2018-eular.5404

SATURDAY, 16 JUNE 2018

\section{Multi-disciplinary management of complex persistent pain}

\section{OP0353-HPR THE EFFECTS OF ARTHRITIS GLOVES ON HAND PAIN IN PEOPLE WITH RHEUMATOID OR INFLAMMATORY ARTHRITIS: A RANDOMISED CONTROLLED TRIAL (A- GLOVES TRIAL)}

A. Hammond ${ }^{1}$, Y. Prior ${ }^{1}$, C. Sutton ${ }^{2}$, S. Cotterill ${ }^{3}$, C. Heal ${ }^{3}$, E. Camacho $^{3}$, on behalf of The A-GLOVES research team. ${ }^{1}$ Centre for Health Sciences Research, University of Salford, Salford; ${ }^{2}$ Lancashire CTU, University of Central Lancashire, Preston; ${ }^{3}$ School of Health Sciences, Manchester University, Manchester, UK

Background: Arthritis (compression) gloves are commonly provided to people with rheumatoid arthritis $(R A)$ and undifferentiated inflammatory arthritis $(I A)$ in the UK health service. These apply pressure and warmth to relieve hand pain, stiffness and improve hand function. A systematic review identified little evidence to support their use.

Objectives: This randomised controlled trial tested effectiveness and cost-effectiveness of mid-finger length compression (intervention) gloves (20\% Lycra: commonest glove model provided) with control gloves (i.e. oedema gloves: $11 \%$ Lycra: fitted at least one size too big) in people with RA and IA.

Methods: Both gloves, which had similar thermal qualities although the control gloves did not provide compression, were provided by rheumatology occupational therapists, following training. ${ }^{2}$ Participants were also given brief advice on hand exercise and joint protection. Adults with RA/IA and persistent hand pain were randomised 1:1 to the two glove types, stratified by disease modifying anti-rheumatic drug (DMARD) change in previous 12 weeks. The primary outcome was dominant hand pain on activity Visual Analogue Scale (VAS:0-10); other outcomes included night hand pain, hand stiffness (both 0-10 VAS); Measure of Activity Performance Hand (MAP-HAND: 0-3). Multiple linear regression was undertaken to estimate the effect of group allocation on hand pain during activity, adjusting for the stratification variable and baseline values. Cost-effectiveness used individual patient level costs (intervention plus healthcare utilisation) and health benefit data (EQ-5D) to calculate costs and QALYs.

Results: 206 participants were randomised (103 to each glove type): median age 59 years [IQR 51,67]; women:166 (81\%); mean disease duration: 8.2 (SD 9.5) years; employed:76 (37\%); right hand dominant:185 (90\%). Of these, $163(79 \%)$ completed 12 week follow-up questionnaires. Both groups reported similar adherence to glove wear (mean 5.2 days/week). At $12 \mathrm{w}$, hand pain scores in both groups similarly improved: the between-groups mean difference of 0.1 was not statistically significant $(95 \% \mathrm{Cl}:-0.47$ to $0.67 ; p=0.72)$. There were no significant differences between groups on any measures, with both groups improving similarly between baseline and $12 \mathrm{w} .73 \%$ in both groups considered gloves beneficial. Intervention gloves had higher costs (£552 (SD £464); control £391 (SD £543) but comparable benefits to control gloves. Intervention gloves would cost $£ 83700$ to gain one QALY and were not likely to be cost-effective.

Conclusions: Compression (intervention) and loose-fitting arthritis (control) gloves had similar effects on hand pain, stiffness and function. Therefore, compression is not the 'active ingredient' in arthritis gloves. Loose fitting gloves providing warmth were perceived as equally effective by participants. We do not know if the therapist effect is important or whether ordinary gloves providing warmth would provide similar results.

\section{REFERENCES:}

[1] Hammond, et al. Clin Rehabil 2016 30:213-24.

[2] Prior, et al. Rheumatology 2017. www.abstractsonline.com/pp8/\#!/4205 